\title{
Revisiones
}

\section{Telemedicina y su aplicación en Dermatología Laboral}

\section{Teledermatology and Ocupational Health}

\author{
M. Victoria Rollón González 1,4, Olga de la Peña Gutiérrez ${ }^{2,4}$, Christian Meier de Taboada ${ }^{3,4}$ \\ 1. Mutua Universal. Madrid. España. \\ 2. Hospital Universitario de Getafe. Madrid. España. \\ 3. Hospital Fundación Alcorcón. Madrid. España. \\ 4. Unidad Docente de Medicina del Trabajo de la Comunidad de Madrid. Madrid. España.
}

Recibido: 23-01-14

Aceptado: 24-02-14

\section{Correspondencia}

Christian Meier de Taboada

Correo electrónico: kurtmeier@hotmail.com

Este trabajo se ha desarrollado dentro del Programa Científico de la Escuela Nacional de Medicina del Trabajo del Instituto de Salud Carlos III en convenio con Unidad Docente de Medicina del Trabajo de la Comunidad de Madrid.

Resumen

Introducción: La importancia y el impacto de la telemedicina han provocado que se aplique a todas las áreas posibles del conocimiento médico y que los tipos de telemedicina hayan crecido de forma paralela al desarrollo de las nuevas tecnologías. La dermatología fue una de las primeras especialidades en la que se aplicaron tecnologías de telemedicina. Las enfermedades de la piel suponen la segunda causa de notificación de enfermedad profesional por lo que aplicar la teledermatología a nivel del ámbito laboral podría ayudar a llevar a cabo un mejor y precoz diagnóstico, al mismo tiempo que podría tener un significativo impacto en ahorro de costes económicos.

Objetivo: Conocer el estado actual de la evidencia en Teledermatología y su potencial traslación al campo de la Medicina del Trabajo, así como identificar el conocimiento y evidencia existentes en los siguientes aspectos críticos que marcan el interés de esta potencial traslación: exactitud diagnóstica, costebeneficio y coste-efectividad, aceptabilidad y satisfacción del paciente, satisfacción del profesional y reducción del tiempo para realizar el diagnóstico

Material y Métodos: Se realiza una revisión bibliográfica de la literatura científica publicada entre 2006-2013. Se consultaron las bases de datos MEDLINE, OSH UPDATE, IBSST,CISDOC, LILACS,WOK, Clinicalkey, Scielo, SCOPUS,OVIDSP, Biblioteca Cochrane e IBECS.

Resultados: Se seleccionaron 13 artículos que incluyen 2 revisiones sistemáticas, 6 ensayos clínicos y 5 estudios transversales. La Teledermatología presenta una exactitud diagnóstica comparable a la consulta convencional, siendo ésta entre un 5\%-11\% más exacta, mostrándose coste-efectiva (1,78 veces más barata) si tenemos en cuenta desplazamientos y pérdida de productividad, con disminución en el tiempo de espera.

Conclusiones: Existen pocas referencias sobre teledermatologia aplicada al ámbito laboral. Teniendo en cuenta la evidencia recogida sobre exactitud diagnóstica, coste-beneficio, aceptabilidad, satisfacción y reducción de tiempos de espera; ésta podría ser una herramienta útil para el Médico del Trabajo que permitiría el acceso a un servicio especializado facilitando un diagnóstico y tratamiento precoz, promoviendo 
la adopción de medidas preventivas, mejorando el seguimiento y evitando ausencias prolongadas del puesto de trabajo, bien por tiempos de consulta o por incapacidad laboral por estudio de enfermedad profesional.

Med Segur Trab (Internet) 2014; 60 (234) 161-178

Palabras clave: "telemedicine", "occupational bealth", "teledermatology", "cost-effectivity"

Summary

Introduction: The importance and impact of telemedicine has caused to be applied to all possible areas and the types of telemedicine have grown in parallel to the development of new technologies. The dermatology was one of the first specialties to adopt the telemedicine. The skin diseases pose a very important issue within the occupational pathology. So applying the teledermatology at workplace level could help to perform better and earlier diagnosis, at the same time that could have a significant impact on economic cost savings.

Objective: Knowing the current state of the evidence in Teledermatology and its potential translation to the field of Occupational Medicine, and to identify existing knowledge and evidence on the following critical issues that set the potential interest of this translation: Diagnostic Accuracy; Cost-benefit and costeffectiveness; Acceptability and patient satisfaction; Professional satisfaction; Reduction of time for diagnosis.

Material and Methods: A literature review of the scientific literature published between 2006-2013 was performed. The MEDLINE, OSH UPDATE, IBSST, CISDOC, LILACS, WOK, ClinicalKey, SciELO, SCOPUS, OVIDSP, IBECS and Cochrane Library were searched.

Results: 13 items including 2 systematic reviews, 6 clinical trials, and 5 cross-sectional studies were selected. The results show that Teledermatology has a diagnostic accuracy comparable to conventional query, this being between 5\% -11\% more accurate. Results also show that Teledermatology is cost-effective (1.78 times cheaper) when you consider displacement and loss of productivity, and decreasing average waiting time for consultancy.

Conclusions: There are few references on Teledermatology applied to the workplace. Given the evidence collected on diagnostic accuracy, cost-effectiveness, acceptability, satisfaction, and reduced waiting times, this could be a useful tool for the Occupational Health because it would allow access to a specialized service providing early diagnosis and treatment, promoting the adoption of preventive measures, improving monitoring, and avoiding prolonged absences from work, due to consultation times or to prolonged labor incapacity while the occupational disease is examined.

Med Segur Trab (Internet) 2014; 60 (234) 161-178

Key words: "telemedicine", "occupational health" and "teledermatology" "cost-efficiency" 


\section{INTRODUCCIÓN}

En términos sencillos telemedicina significa "medicina a distancia", del griego tele ("a la distancia"), aunque varias son las definiciones dadas por distintas organizaciones e instituciones con competencia en esta materia.

La Organización Mundial de la Salud, define la telemedicina como "el suministro de servicios de atención sanitaria, en los que la distancia constituye un factor crítico, por profesionales que apelan a las tecnologías de la información y de la comunicación con objeto de intercambiar datos para hacer diagnósticos, preconizar tratamientos y prevenir enfermedades y accidentes, así como para la formación permanente de los profesionales de atención de salud y en actividades de investigación y evaluación, con el fin de mejorar la salud de las personas y de las comunidades en que viven" ${ }^{1}$

Otra de las definiciones es la que propone la American Telemedicine Association, que considera la telemedicina como el intercambio de información médica de un lugar a otro, usando las vías de comunicación electrónicas, para la salud y educación del paciente o el proveedor de los servicios sanitarios y con el objetivo de mejorar la asistencia del paciente $^{2}$.

Actualmente, este concepto se ve ampliado para incluir la transferencia de recursos sanitarios y de cuidados de salud de todo tipo, pasando a denominarse e-Salud (e-health). La Organización Mundial de la Salud (OMS) indica que la e-salud incluye tres áreas: la distribución de información sanitaria por medios electrónicos; el uso de las tecnologías de la información y la comunicación (TIC) y el e-commerce para mejorar los servicios de salud pública; y el uso de e-business en sistemas de gestión de la salud. La e-salud (e-bealth) quedaría así definida como el uso de las tecnologías de la información y telecomunicación para conocer las necesidades de los ciudadanos, pacientes, profesionales de la salud, proveedores de servicios en salud y legisladores con respecto a la prestación de los diferentes servicios en salud, mientras que la telemedicina es el uso de las telecomunicaciones para diagnosticar y tratar enfermedades ${ }^{3}$.

El concepto de telemedicina implica la existencia de un emisor, proveedor de información y un receptor que interactúan mediante el uso de las tecnologías de información y telecomunicación. Es decir, se envía una información que el receptor procesa, enviando la contestación o diagnóstico.

Tres son las ventajas básicas de la telemedicina:

- Mejora del acceso a los servicios para poblaciones distantes.

- Disminución de los costes en desplazamiento de pacientes y médicos.

- Facilitación de la difusión y puesta al día de los conocimientos médicos.

La importancia y el impacto de la telemedicina ha provocado que se aplique a todas las áreas posibles y que los tipos de telemedicina hayan crecido de forma paralela al desarrollo de las nuevas tecnologías, de los distintos tipos de telemedicina: teleconsulta, teleeducación, telemonitorización, o telecirugía, es la primera la de mayor uso en la actualidad.

La generalización del uso de los servicios de telemedicina es una gran oportunidad para los profesionales de la salud y los ciudadanos para mejorar la salud, al mismo tiempo que puede tener un significativo impacto en ahorro de costes económicos, haciendo que los sistemas de salud pública sean más democráticos, accesibles y viables. Algunos estudios de análisis de coste-beneficio muestran beneficios potenciales de hasta el $70 \%$ en ahorro de costes, teniendo en cuenta todos los factores asociados (inversión directa en infraestructura, costes operacionales, de profesionales, etc.) y beneficios (calidad, eficiencia, acceso, etc.) para los ciudadanos, organizaciones de salud, aseguradoras, etc. En Europa, por ejemplo, se espera que el mercado de la "tele atención" genere más de cinco mil millones de euros/año para $2015^{4}$. Actualmente, el mercado de E-salud es aproximadamente el $2 \%$ del gasto total de salud, pero tiene potencial para doblarse hasta alcanzar casi el volumen del gasto en aparatos médicos y la mitad del gasto en farmacia. 
Este impacto es especialmente importante en países en desarrollo, donde se entiende la telemedicina como una oportunidad para dar a gran parte de la población acceso a la salud a un coste razonable. Debido a ello, el número de nuevos proyectos asociados con la telemedicina dentro del campo de la salud han crecido de un 8\% en el periodo1991- 1995 a un 43\% en el periodo 2006- 2011 (Center for Health Markect Innovations) ${ }^{5}$,

Sin embargo, a pesar de la existencia de un nivel de madurez general en las aplicaciones de telemedicina en los Sistemas Públicos de Salud y la existencia de dispositivos que permiten dar atención personalizada independientemente de la localización de los pacientes, el mercado no se ha desarrollado todavía a gran escala. Además, la transferencia de soluciones entre distintos países y la posibilidad de inter operar entre ellos sigue siendo una excepción. Debido a ello, la Unión Europea ha lanzado una iniciativa muy ambiciosa en su programa Horizonte 2020, en el que la e-salud cuenta con un apartado completo y la aplicación de las técnicas de telemedicina para el diagnóstico remoto y la atención a ancianos y discapacitados desde el hogar. Proyectos como RenewingHealth o Nexes son buena muestra de ello ${ }^{6,7}$.

La dermatología fue una de las primeras especialidades en adoptar la telemedicina ya que es una especialidad predominantemente visual y la imagen es el estándar de oro para el diagnóstico de las patologías cutáneas. El diagnóstico y seguimiento es posible pues, si las imágenes son de buena calidad.

Se distinguen dos tipos de Teledermatología ${ }^{8}$ (TD): la Teledermatología en tiempo real (TDTR) también llamada síncrona y la Teledermatología Asíncrona (TDA) también llamada "de almacenamiento", diferida o store-and-forward.

En la TDTR el dermatólogo observa la imagen del paciente en el mismo momento en que es captada, y realiza un diagnóstico inmediato. En la TDA se registran imágenes del paciente en un momento determinado, y se transfieren al dermatólogo, el cual emite un diagnóstico y manejo del paciente en tiempo diferido. La mayoría de los dermatólogos se inclinan a utilizar la forma asíncrona o diferida debido a que es más sencilla, genera menor consumo de recursos informáticos y es de menor coste.

La Asociación Americana de Telemedicina (ATA) ha establecido una guía para la práctica de la teledermatología ${ }^{9}$ con el fin de ayudar al desarrollo de prácticas armonizadas, seguras y eficaces.

Amplios estudios avalan la fiabilidad de la TD, si entendemos ésta como concordancia diagnóstica entre dermatología clínica y TD sin verificación histopatológica, con un grado de recomendación A para lesiones dermatológicas en general ${ }^{10,11,12,13,14}$. Si evaluamos distinción entre malignidad y benignidad la TD, es una técnica con una fiabilidad excelente con un grado de recomendación $\mathrm{B}$, obteniendo los mejores resultados de concordancia interobservador ${ }^{10 .}$

Las enfermedades de la piel suponen un problema de salud importante dentro de la patología laboral. Las dermatosis profesionales adquieren cada día mayor importancia.

El análisis de los datos de enfermedades profesionales (CEPROSS) recibidos desde Enero de 2013 hasta Noviembre de 2013 por el Observatorio de Enfermedades Profesionales de la Seguridad Social, confirma que las enfermedades profesionales correspondientes al GRUPO 5, ocuparían el segundo lugar en partes notificados con 869 partes. A los que habría que añadir los cuadros dermatológicos producidos por agentes incluidos en otros $\operatorname{grupos}^{15,16,17,18}$. De los 869 casos notificados en este periodo, 394 ocasionaron baja por dermatosis profesional.

A nivel global, en el periodo de estudio de las enfermedades profesionales (2007-2012), la duración media en días de baja de este grupo 5 fue de 57,45 días, superando en 7,45 días el tiempo medio de baja por enfermedad profesional. En relación a la distribución por edades, las dermatosis profesionales afectan a trabajadores más jóvenes (38,4 años) frente al grupo de edad más prevalente de la enfermedad profesional (40-49 años). 
Dado el impacto de las enfermedades profesionales de la piel, tanto por su relevancia con un segundo lugar en frecuencia de partes comunicados a CEPROSS, como por el incremento de costes ocasionados por los periodos de incapacidad laboral, como por su por su influencia en la calidad de vida del trabajador, teniendo en cuenta el factor agravante de afectación a trabajadores en edad joven, con una pérdida añadida para el desempeño laboral, sería importante el diagnóstico precoz de las dermatosis de origen profesional así como un buen manejo y tratamiento.

La implementación de la Teledermatología dentro del ámbito laboral podría mejorar el acceso a las consultas dermatológicas y el contacto con profesionales Especialistas en Dermatología que podrían ayudar a llevar a cabo un mejor y precoz diagnóstico de las dermatosis profesionales.

Nos planteamos, por tanto, como objetivo general conocer el estado actual de la evidencia en Teledermatología y su potencial traslación al campo de la Medicina del Trabajo.

Teniendo como objetivos específicos identificar el conocimiento y evidencia existentes en los siguientes aspectos críticos que marcan el interés de esta potencial traslación: Exactitud diagnóstica, Coste-beneficio y coste-efectividad, aceptabilidad y satisfacción del paciente, Satisfacción del profesional y Reducción del tiempo para realizar el diagnóstico.

\section{METODOLOGÍA}

Se realizó una búsqueda bibliográfica en las bases de datos MEDLINE (vía PubMed), OSH UPDATE, IBSST,CISDOC, LILACS,WOK, OVID-SP,OECD, SCOPUS, WOK, Clinicalkey, Biblioteca Cochrane e IBECS. La búsqueda se completó con literatura científica obtenida en el repositorio SciELO, Google y así como artículos referenciados en los trabajos que se incluyeron en el estudio.

Se definieron diferentes estrategias de acuerdo a los motores de búsqueda de cada base de datos utilizando Descriptores "MeSH" y términos libres que figuran en la tabla I.

Tabla I. Bases de datos y ecuación de búsqueda empleados

\begin{tabular}{|c|c|}
\hline Bases de datos & $\begin{array}{c}\text { TÉRMINOS DE BÚSQUEDA: MESH } \\
\text { "telemedicine", “occupational health" y "teledermatology” }\end{array}$ \\
\hline $\begin{array}{l}\text { CISDOC - Base de Datos de Salud y Seguridad } \\
\text { en el trabajo (OIT) } \\
\text { http://www.ilo.org/safework/info/publications/ } \\
\text { WCMS_112523/lang--en/index.htm }\end{array}$ & "teledermatologia" OR "teledermatology" \\
\hline $\begin{array}{l}\text { Clinicalkey - ELSAVIER } \\
\text { https://www.clinicalkey.com/\#!/SearchCtrl/ } \\
\text { doSearchResults/telemedicine/// }\end{array}$ & "telemedicine" AND "teledermatology" \\
\hline $\begin{array}{l}\text { COCHRANE LIBRARY Plus - Biblioteca Cochrane } \\
\text { Plus en Español } \\
\text { http://www.update-software.com/Clibplus/ClibPlus. } \\
\text { asp }\end{array}$ & ((TELEMEDICINE) AND (TELEDERMATOLOGY)):TA \\
\hline $\begin{array}{l}\text { Global Health (OVIDSP) - Public Health } \\
\text { and Tropical Medicine y Cab Abstracts } \\
\text { http://ovidsp.ovid.com/autologin.cgi }\end{array}$ & $\begin{array}{l}\text { ((TELEMEDICINE) AND (TELEDERMATOLOGY) } \\
\text { AND (OCCUPATIONAL HEALTH)) }\end{array}$ \\
\hline $\begin{array}{l}\text { IBECS - Índice Bibliográfico Español en Ciencias } \\
\text { de la Salud } \\
\text { http://ibecs.isciii.es }\end{array}$ & "telemedicine" AND "teledermatology" \\
\hline $\begin{array}{l}\text { IBSST - Índice Bibliográfico de Salud } \\
\text { y Seguridad en el Trabajo } \\
\text { http://ibsst.isciii.es/ }\end{array}$ & "teledermatologia" OR "teledermatology" \\
\hline
\end{tabular}




\begin{tabular}{|c|c|}
\hline Bases de datos & $\begin{array}{c}\text { TÉRMINOS DE BÚSQUEDA: MESH } \\
\text { "telemedicine", "occupational health" } y \text { "teledermatology" }\end{array}$ \\
\hline $\begin{array}{l}\text { LILACS - Literatura Latinoamericana y del Caribe } \\
\text { en CC de la Salud } \\
\text { http://bases.bireme.br/cgi-bin/wxislind.exe/iah/ } \\
\text { online/?IsisScript=iah/iah.xis\&base=LILACS\&lang=e }\end{array}$ & "telemedicine" AND "teledermatology" \\
\hline $\begin{array}{l}\text { Scielo - España } \\
\text { http://scielo.isciii.es/scielo.php }\end{array}$ & $\begin{array}{l}\text { ( teledermatologia ) or TELEDERMATOLOGY [Todos } \\
\text { los indices] and (telemedicina) or TELEMEDICINE } \\
\text { [Todos los indices] }\end{array}$ \\
\hline $\begin{array}{l}\text { OECD HealthStatistics } \\
\text { http://www.oecd-ilibrary.org/social-issues- } \\
\text { migration-health/data/oecd-health-statistics_health- } \\
\text { data-en }\end{array}$ & "telemedicine" AND "teledermatology" \\
\hline $\begin{array}{l}\text { OSH UPDATE - Occupational Safety and Health } \\
\text { http://www.oecd-ilibrary.org/social-issues- } \\
\text { migration-health/data/oecd-health-statistics_health- } \\
\text { data-en }\end{array}$ & "teledermatology" \\
\hline $\begin{array}{l}\text { PubMed - Acceso público a las bases de datos } \\
\text { de la NLM } \\
\text { http://www.ncbi.nlm.nih.gov/entrez/query/fgci }\end{array}$ & $\begin{array}{l}((((((((((\text { "Workplace”[Mesh]) OR "Occupational } \\
\text { Health Services"[Mesh]) OR "Occupational } \\
\text { Health”[Mesh]) OR "Occupational Exposure"[Mesh]) } \\
\text { OR "Accidents, Occupational”[Mesh]) OR } \\
\text { "Occupational Diseases"[Mesh]) OR "Occupational } \\
\text { Medicine”[Mesh])) AND “Telemedicine"[Mesh]))) } \\
\text { AND teledermatology }\end{array}$ \\
\hline $\begin{array}{l}\text { SCOPUS } \\
\text { http://www.scopus.com/ }\end{array}$ & "telemedicine" AND "teledermatology" \\
\hline $\begin{array}{l}\text { Web of Knowledge - (WOK) } \\
\text { http://www.accesowok.fecyt.es/login/ }\end{array}$ & $\begin{array}{l}\text { "telemedicine" AND "teledermatology" } \\
\text { Topic=(teledermatology) AND Topic=(telemedicine) } \\
\text { AND Topic=(occupational health) }\end{array}$ \\
\hline
\end{tabular}

Para la selección de los artículos a incluir en la revisión se establecieron los criterios de selección que figuran en la siguiente tabla.

Tabla II. Los criterios de inclusión y exclusión

\begin{tabular}{|c|c|}
\hline Criterios de inclusión: & Criterios de exclusión: \\
\hline \multirow{2}{*}{$\begin{array}{l}\text { - Fecha de publicación entre enero de } 2006 \text { y di- } \\
\text { ciembre de } 2013 \text {. }\end{array}$} & $\begin{array}{l}\text { - Artículos referentes únicamente a la valoración de } \\
\text { los equipos utilizados. }\end{array}$ \\
\hline & $\begin{array}{l}\text { - La teledermatología como herramienta de apren- } \\
\text { dizaje para médicos de atención primaria y resi- } \\
\text { dentes. }\end{array}$ \\
\hline \multirow[b]{2}{*}{ — Estudios publicados en español e inglés. } & - Comentarios, editoriales u opiniones de expertos. \\
\hline & $\begin{array}{l}\text { - Artículos destinados a evaluar la fiabilidad diag- } \\
\text { nostica mediante teledermatología. }\end{array}$ \\
\hline \multirow{2}{*}{$\begin{array}{l}\text { - Artículos cuyo objeto principal de estudio dentro } \\
\text { de la telemedicina fuera un aspecto relacionado } \\
\text { con la teledermatología. }\end{array}$} & — Estudios en menores de 16 años. \\
\hline & - Artículos duplicados. \\
\hline
\end{tabular}

Para determinar la evidencia científica de cada artículo, se evaluó con los criterios SIGN de la "Scottish Intercollegiate Guidelines Network"19. 


\section{RESULTADOS}

A partir de la estrategia de búsqueda se obtuvo una relación con un total de 167 artículos, a los que se aplicaron los criterios de inclusión y exclusión quedando un total de 38 artículos. Se eliminaron los duplicados quedando 33 artículos, que tras su revisión a texto completo y evaluar su pertinencia en función de los objetivos del estudio se incluyeron un total de 13 artículos.

Las posibles discordancias se solucionaron mediante consenso entre los componentes del equipo.

Se encontraron dos revisiones sistemáticas con un nivel de evidencia de $1+$, seis ensayos clínicos de ellos uno con evidencia 1++, tres con 1+ y dos con evidencia 1-; se encontraron cinco estudios transversales con evidencia 3.

De los 13 artículos: 5 se relacionan con la exactitud diagnóstica, sensibilidad y especificidad, 5 con costes, 3 con satisfacción de los pacientes y 1 con satisfacción del personal sanitario que solicitaba y realizaba la teleconsulta.

Las diferentes aplicaciones clínicas de la teledermatología son evaluadas mediante una revisión sistemática por Moreno-Ramírez D; et al. 2008 $^{20}$, considerando los siguientes aspectos de la teledermatologia: herramienta diagnóstica con análisis de calidad, validez y fiabilidad; efectividad como sistema asistencial, evaluación económica y satisfacción con la asistencia mediante la teledermatología.

Se valoran las distintas modalidades de teledermatología: diferida, a tiempo real, mediante telefonía móvil, teledermatoscopía y asistida por el paciente.

Desde 1998 hasta Julio de 2006 se capta la población atendida mediante cualquier modalidad de telemedicina y los profesionales usuarios de la misma.

Se revisan un total de 32 estudios $(n=32)$ : observacionales $(n=21)$, aleatorizados controlados $(n=9)$ y cuasi-experimentales $(n=2)$. La TD aplicada a cuadros dermatológicos, demuestra una exactitud diagnóstica entre 59\% - 89\%. La TDTR ha demostrado una sensibilidad entre $74 \%-97 \%$, con una especificidad del $86 \%$ al $100 \%$. Concluyen que la TDTR y la TDA presentan una exactitud buena; mientras que la TD asistida por el paciente presentaría una validez adecuada. La TD en el diagnóstico de cáncer de piel presenta una concordancia con el método de referencia elevada, con un índice kappa de 0,79, y un porcentaje de coincidencia del $48 \%$ al $100 \%$.

La teledermatoscopia ha demostrado una validez excelente, alcanzando una sensibilidad de 1 y una especificidad de 0,78 . El índice de concordancia (kappa) oscila entre 0,74 y 0,94 con un acuerdo global del $86 \%$; respecto a la consulta presencial. La TDA entre dermatólogo y atención primaria es más valida que la TD basada en la transmisión de imágenes en el screening de cáncer de piel.

La fiabilidad de la TD, alcanzó un 64\% de acuerdo respecto al método de referencia para cuadros dermatológicos, con un $79 \%$ de acuerdo y kappa de 0,75 .

Los mejores resultados de fiabilidad de la TD corresponden a TD aplicada en cáncer de piel con índice kappa entre 1 y $0,91$.

Se demuestra la efectividad de la TD con una disminución del 18,5\% al 60,7\% de las consultas hospitalarias. La TDA disminuye significativamente la demora para la intervención; respecto a la consulta convencional.

La TDA es más coste-efectiva considerando el descenso de la demora asistencial.

Los resultados sobre el grado de satisfacción reflejan que entre el $79 \%$ y el 90\% de los pacientes están satisfechos con la TD, entre médicos usuarios del $21 \%$ al $92 \%$ se encuentran satisfecho y los dermatólogos que utilizan la TD presentan resultados de satisfacción del $71,2 \%$ al $75 \%$. 
Warshaw, Erin M. et al. $2011^{21}$ Realizan una revisión sistemática de 78 artículos publicados en lengua inglesa entre 1990 y 2009.

El objetivo de identificar la evidencia relacionada con los siguientes aspectos:

1a Exactitud diagnóstica de la TD comparada con la consulta presencial, 1b Concordancia diagnóstica entre TD y consulta presencial, 2a Exactitud de la TD en el manejo de las lesiones comparada con la consulta presencial. 2b Concordancia en el manejo de las lesiones entre TD y consulta presencia. 3 Resultados clínicos (curso clínico, satisfacción, calidad de vida, visitas evitadas) de la TD comparada con la consulta presencial. 4 Coste de la TD comparada con la consulta presencial.

Para las cuestiones 1 y 2 se evaluaron 51 artículos. Para 1a se concluyó que la consulta presencial es entre un 5 y 11\% más exacto para diagnóstico primario. Al utilizar teledermatoscopia se observan mejores resultados para lesiones aisladas. 1b. la concordancia diagnóstica para TDA era de un 64\%, aumentando hasta un $87 \%$ para TDTR. 2a Sólo 2 estudios informaban de esta cuestión por lo que es difícil de generalizar resultados, aunque se observaron tasas equivalente (Diferencias absolutas de $+-10 \%$ ). $2 \mathrm{~b}$ Las tasas de concordancia en cuanto al manejo de las lesiones era entre buena y muy buena (kappas comprendidos entre 0,62 y 0,82). 3. Curso clínico: 3 estudios que no muestran suficiente evidencia para demostrar el efecto de la TD sobre el curso clínico. Satisfacción del paciente: 11 estudios. Satisfacción global con la TD muy alta, entre el 79 y el 86\%. Preferencias del paciente: 12 estudios. Amplia variación en cuanto a la preferencia por la TD, entre el 36 y el $86 \%$, con aumento de la aceptación en caso de esperas muy largas para acudir a la consulta presencial (entre 68 y 76\%). Tiempo de tratamiento: 4 estudios. Rango de días de espera para inicio de tratamiento 44 en TD frente a 76,3 días en consulta presencial ( $\mathrm{p}<0.001)$, y de 19 días menos para la biopsia $(\mathrm{p}=0.03)$. Visitas evitadas: 14 estudios. Amplia variación (disminución entre 12,8\% y 72\%) dependiendo del tipo de lesión. 4 Coste: 10 estudios. La TD es coste-efectiva si se tienen en cuenta los costes directos e indirectos, siendo muy importante factores como distancia de desplazamiento o pérdida de productividad. Los autores concluyeron que en general la consulta clínica era más exacta en cuanto al diagnóstico y al manejo que la TD, asumiendo que en ambos casos los servicios eran prestados por dermatólogos, también indicaron que los resultados eran altamente dependientes de la instalación y formación del personal que llevaba a cabo la consulta de TD. También concluyeron que los resultados de la TD pueden ser superiores a la consulta clínica si ésta se lleva a cabo por médicos no especializados en dermatología.

Tabla III. Revisiones sistemáticas

\begin{tabular}{|c|c|c|c|c|c|c|c|}
\hline Autor & $\begin{array}{l}\text { Año / } \\
\text { País }\end{array}$ & Muestra & Colectivo & Efecto estudiado & Resultado & $\begin{array}{c}\text { Control } \\
\text { factor de } \\
\text { confusión }\end{array}$ & $\begin{array}{c}\text { Nivel de } \\
\text { evidencia }\end{array}$ \\
\hline $\begin{array}{l}\text { Moreno- } \\
\text { Ramírez, } \\
\text { D. }{ }^{20}\end{array}$ & $\begin{array}{l}2008 / \\
\text { España }\end{array}$ & $\begin{array}{l}32 \text { estudios: } \\
\text { observacionales } \\
(n=29), \\
\text { aleatorizados } \\
\text { controlados } \\
(n=9), \text { cuasi- } \\
\text { experimentales } \\
(n=2)\end{array}$ & $\begin{array}{l}\text { Pacientes Atendidos } \\
\text { Mediante Cualquier } \\
\text { Modalidad De } \\
\text { Teledermatología } \\
\text { Y Profesionales } \\
\text { Sanitarios Usuarios } \\
\text { De Alguna De } \\
\text { Éstas. Periodo: } 1998 \\
\text { Hasta Julio } 2006\end{array}$ & $\begin{array}{l}\text { Analiza la Validez, } \\
\text { Fiabilidad, } \\
\text { Efectividad Clínica } \\
\text { o Asistencial, } \\
\text { Resultado } \\
\text { Económico, Valores } \\
\text { De Satisfacción } \\
\text { De Pacientes Y } \\
\text { Usuarios De TD }\end{array}$ & $\begin{array}{l}\text { La TD Presenta } \\
\text { buena Validez } \\
\text { (Nivel Evidencia } \\
\text { Ib, Recomendación } \\
\text { A), Fiabilidad } \\
\text { Adecuada(Nivel } \\
\text { Evidencia Ib, } \\
\text { Recomendación A), } \\
\text { Efectividad Buena } \\
\text { (Nivel Evidencia Ib, } \\
\text { Recomendación B), } \\
\text { Satisfacción Elevado } \\
\text { (Nivel Evidencia Ib, } \\
\text { recomendación A) }\end{array}$ & SI & $1+$ \\
\hline
\end{tabular}




\begin{tabular}{|c|c|c|c|c|c|c|c|}
\hline Autor & $\begin{array}{c}\text { Año / } \\
\text { País }\end{array}$ & Muestra & Colectivo & Efecto estudiado & Resultado & $\begin{array}{c}\text { Control } \\
\text { factor de } \\
\text { confusión }\end{array}$ & $\begin{array}{l}\text { Nivel de } \\
\text { evidencia }\end{array}$ \\
\hline $\begin{array}{l}\text { Warshaw, } \\
\text { Erin } \text { M. }^{21}\end{array}$ & $\begin{array}{l}2011 / \\
\text { EE.UU. }\end{array}$ & 78 artículos & $\begin{array}{l}\text { Criterios Inclusión, } \\
\text { Exclusión Extensos } \\
\text { Trabajos Entre } 1990 \\
\text { Y } 2009\end{array}$ & $\begin{array}{l}\text { Análisis de } \\
\text { exactitud y } \\
\text { concordancia en } \\
\text { el diagnóstico } \\
\text { y manejo entre } \\
\text { consulta presencial } \\
\text { y TD. Seguimiento } \\
\text { clínico y costes }\end{array}$ & $\begin{array}{l}\text { Exactitud } \\
\text { diagnóstica } \\
\text { diferencia en } \\
\text { porcentajes } \\
\text { absolutos entre FTF } \\
\text { y STF (19\% y un 5\% } \\
\text { dependiendo tipo } \\
\text { de lesión) tasas } \\
\text { equivalentes para } \\
\text { la exactitud en } \\
\text { el manejo de las } \\
\text { lesiones. Rango } \\
\text { de preferencia } \\
\text { por TD (38-86\%) } \\
\text { tasas comparables } \\
\text { de satisfacción. } \\
\text { Tiempo de espera } \\
\text { más cortó con TD. } \\
\text { La mayoría de los } \\
\text { estudios encuentran } \\
\text { que la TD es coste } \\
\text {-efectiva, sobre } \\
\text { todo si se tiene en } \\
\text { cuenta la lejanía del } \\
\text { paciente. }\end{array}$ & SI & $1+$ \\
\hline
\end{tabular}

Bowns, IR. est al. 2006 ${ }^{22}$ llevan a cabo un ensayo en ocho consultorios de medicina general y el servicio de dermatología de un Hospital. Uno de los objetivos fue evaluar la equivalencia entre la TDA y la consulta presencial, así como valorar la opinión de los pacientes y clínicos sobre el servicio. Se realizó un ensayo prospectivo randomizado controlado, seleccionando adultos (mayores de 16 años) que acudieron a atención primaria para una primera consulta por problemas dermatológicos, estos pacientes fueron remitidos a dermatología previa toma de contacto y realización de fotografías digitales de las lesiones dérmicas y se tomó un grupo control que fue remitido directamente al especialista. Los resultados no fueron significativos por las dificultades de reclutamiento, la perdida de casos y la demora en obtener la segunda consulta.

El segundo de los objetivos del estudio fue evaluar la equivalencia del uso de la fotografía y dermatoscopia digitales por TD con la consulta presencial en el manejo de casos sospechosos de lesiones malignas (melanoma, carcinoma de células escamosas). Se tomaron a los adultos de las consultas de primaria que consultaron por lesiones dérmicas sospechosas de malignidad, a los que se realizaron series de fotografías digitales y dermatoscópicas de las lesiones sospechas, los pacientes fueron inmediatamente valorados de forma presencial por un dermatólogo y luego valorado también de forma presencial por un segundo especialista quien también valoró las imágenes digitales y ambos diagnósticos son comparados con el diagnóstico definitivo (clínico o histopatológico definitivo). La concordancia entre las dos formas fue de $68 \%$, es decir altamente sensible (98\%, 95\% CI: de 92-99\%) con unos valores especificidad (43\%, 95\% CI: 36-51\%). Un 30\% de los casos no requirieron de consulta presencial para el diagnóstico. Para este segundo objetivo el estudio concluye que es poco probable que la TD pueda reducir la necesidad de las consultas clínicas convencionales y seguir manteniendo al mismo tiempo la seguridad clínica en el diagnóstico.

La concordancia diagnóstica es analizada por Romero, G. et al. $2006^{23}$ en un ensayo aleatorizado prospectivo de concordancia diagnóstica que compara las modalidades de TDTR y TDA. La muestra fue de 159 pacientes remitidos desde atención primaria. En la primera consulta fueron aleatorizados en tres ramas: teledermatología asíncrona (TDA), síncrona (TDTR) y consulta convencional (CC) como grupo control. Para la TDA se utilizó 
imágenes digitales fijas de alta calidad. Los pacientes TDTR fueron evaluados además mediante videoconferencia y todos los pacientes fueron atendidos en consulta presencial considerada como estándar oro. Un segundo dermatólogo evaluó la concordancia diagnóstica y de manejo entre las teleconsultas y las consultas presenciales.

Los 147 pacientes que completaron el estudio, fueron distribuidos como 74 TDA, 47 TDTR y 26 CC (grupo control). la evaluación TD fue idéntica a la de presencial (no error) en 100 pacientes (82,6\%). Los errores fueron leves en $14(11,6 \%)$ y graves en 7 (5,8\%). La concordancia diagnóstica fue muy alta (kappa $=0,813)$. La conclusión del estudio es que la fiabilidad de la TD es muy elevada, especialmente para patología tumoral o infecciosa. La interacción síncrona con audio consume más recursos, es difícil de coordinar y no es más eficaz en diagnóstico que la TDA.

Pak, Hon S. et al. $2009^{24}$ realizan un análisis de coste de la consulta por TDA, comparada con la consulta de dermatología convencional El estudio se llevó a cabo en el Departamento de Defensa de los Estados Unidos de América (DoD), los sujetos del estudio fueron asignados al azar entre las consultas de TD y la convencional. Los costes directos de atención médica se estimaron mediante la combinación de los datos de utilización, de las tasas de reembolso del seguro Medicare y los precios de los medicamentos al por mayor.

Los costes indirectos se calcularon sobre la base de la pérdida de productividad. La contabilización de la pérdida de productividad es definida por la Comisión Técnica de Coste-Efectividad en Salud y Medicina (un promedio de 4 horas de productividad perdida que serían necesarias para asistir a la visita y viajar hacia y desde la visita).

Los costes totales y medios se compararon entre los grupos. En total el coste de los pacientes atendidos mediante teledermatología fue de 119.402 dólares ( $\$ 340$ promedio) y los pacientes atendidos en consulta convencional fue de \$129.133 (\$372 promedio). Desde la perspectiva económica del Departamento de Defensa, la teledermatología asíncrona fue una estrategia de ahorro de costes para la prestación de atención de dermatología en comparación con los métodos convencionales de consulta cuando se incluyen los costes por pérdida de productividad.

Van der Heijden J P; et al. $2011^{25}$ Estudio realizado entre los meses de Marzo de 2007 y Septiembre de 2010; en Holanda.

Participan: 1820 Médicos de Atención Primaria y 166 Dermatólogos, realizándose 37207 teleconsultas.

Se realizan fotografías digitales en consulta de Atención Primaria, enviándose para valoración por Dermatólogos colaboradores, valorándose parámetros de: eficiencia, calidad y costes.

Las teleconsultas resultan eficientes ante la disminución del $74 \%$ de derivaciones a especialistas en Dermatología. Confirman la calidad de la TD, precisando sólo una segunda consulta en $29 \%$ de los casos, objetivándose una reducción de costes del 18\% respecto a la consulta convencional.

La concordancia entre diagnóstico mediante consulta presencia y teleconsulta es también analizada por Ríos-Yuil, J.M. et al. $2011^{26}$ mediante un estudio cuasiexperimental, aleatorizado, en el que se establecieron dos grupos de dermatólogos al azar (Ateneo presencial y Teleateneo) a los que se les expusieron 30 casos clínicos determinándose el grado de correlación (kappa de Cohen). Se utilizaron las decisiones del ateneo presencial como patrón oro. La capacitación entre los dos grupos era homogénea.

Los resultados mostraron un porcentaje de acuerdo del 83,33\%, con un índice kappa $=0,6512$. Se descartaron diferencias interobservador dado el alto grado de correlación entre los grupos. 
Börve, Alexander. et al. $2013^{27}$ realiza un ensayo dirigido a comprobar la exactitud del diagnóstico mediante una aplicación para móvil (app) que permitía enviar imágenes de dermatoscopia desde el propio dispositivo, para ello analizó la concordancia entre el diagnóstico basado en la imagen tomada con este dispositivo con respecto a la consulta presencial, verificó la adecuación de las decisiones de los dermatólogos y evaluó la calidad de las imágenes obtenidas.

Se estudiaron 69 lesiones, correspondientes a 62 pacientes, todos ellos precisaron biopsia o cirugía. El diagnóstico histopatológico se tomó como patrón oro de diagnóstico.

La imagen dermatoscópica junto con la información clínica fue evaluada mediante consulta presencial y de forma diferida por dos teledermastoscopistas.

La exactitud diagnóstica de la consulta presencial, frente al diagnóstico histopatológico, fue del $66,7 \%$, estadísticamente más alta que la obtenida por el primer teleobservador (TD1) $(50,7 \%)$, pero similar al segundo (TD2) $(60,9 \%)$.

En cuanto a la diferenciación entre lesiones benignas y malignas la consulta presencial tuvo una tasa de exactitud del 87\% frente al 75,4 del TD1 y al 79,7 del TD2, sin embargo los resultado en cuanto a la diferenciación de melanocíticos y no melanocíticos fueron prácticamente similares.

La concordancia interobservador mostraban acuerdo moderado, $\mathrm{k}=0,5$ para todos los caso de diagnóstico primario, elevándose a $\mathrm{k}=0,8$ entre melanocíticos y no melanocíticos

Los teledermatoscopistas proporcionaron decisiones adecuadas de manejo clínica para $68(98,6 \%)$ y 69 (100\%) lesiones.

La calidad de la imagen fue citada como excelente o suficiente en 94\% por TD1 y el $84 \%$ TD2.

El estudio puede haber incurrido en un sesgo del observador ya que ambos deteledermatoscopistas sabían que las lesiones habían precisado biopsia o cirugía lo que podía afectar a su decisión de tratamiento.

La ventaja de este sistema es que la aplicación no necesita conexiones complicadas ni equipos complementarios, puesto que el dermatoscopio se conecta al teléfono móvil.

Además la estandarización del procedimiento minimiza el riesgo de que el personal no especializado olvide incluir información relevante.

La conclusión final es que esta aplicación de teledermatoscopia móvil puede ser útil para despistaje de lesiones sospechosas de malignidad en pacientes enviados a consulta de dermatología.

Tabla IV. Ensayos clínicos

\begin{tabular}{|c|c|c|c|c|c|c|c|}
\hline Autor & $\begin{array}{l}\text { Año / } \\
\text { País }\end{array}$ & Muestra & Colectivo & Efecto estudiado & Resultado & $\begin{array}{c}\text { Control } \\
\text { factor de } \\
\text { confusión }\end{array}$ & $\begin{array}{c}\text { Nivel de } \\
\text { evidencia }\end{array}$ \\
\hline $\begin{array}{l}\text { Bowns, } \\
\text { IR }^{22}\end{array}$ & $\begin{array}{c}2006 \text { / } \\
\text { Inglaterra }\end{array}$ & $\mathrm{N}=208$ & $\begin{array}{l}\text { Pacientes de } \\
\text { ocho prácticas de } \\
\text { medicina general y } \\
\text { del departamento } \\
\text { de dermatología } \\
\text { del hospital } \\
\text { en Sheffield, } \\
\text { Inglaterra. }\end{array}$ & $\begin{array}{l}\text { Analiza la } \\
\text { equivalencia } \\
\text { clínica de la } \\
\text { consulta de TDA } \\
\text { con CC utilizando } \\
\text { fotografía digital } \\
\text { y dermatoscopio } \\
\text { en el manejo } \\
\text { de melanoma } \\
\text { o carcinoma de } \\
\text { células escamosas. }\end{array}$ & $\begin{array}{l}\text { Para el segundo } \\
\text { objetivo concluye } \\
\text { que es poco } \\
\text { probable que la } \\
\text { TD pueda reducir } \\
\text { drásticamente } \\
\text { la necesidad de } \\
\text { consultas clínicas } \\
\text { convencionales, } \\
\text { manteniendo al } \\
\text { mismo tiempo la } \\
\text { seguridad clínica. }\end{array}$ & No & 1- \\
\hline
\end{tabular}




\begin{tabular}{|c|c|c|c|c|c|c|c|}
\hline Autor & $\begin{array}{l}\text { Año / } \\
\text { País }\end{array}$ & Muestra & Colectivo & Efecto estudiado & Resultado & $\begin{array}{l}\text { Control } \\
\text { factor de } \\
\text { confusión }\end{array}$ & $\begin{array}{l}\text { Nivel de } \\
\text { evidencia }\end{array}$ \\
\hline $\begin{array}{l}\text { Romero, } \\
\text { G. }{ }^{23}\end{array}$ & $\begin{array}{l}2006 \text { / } \\
\text { España }\end{array}$ & $N=159$ & $\begin{array}{l}\text { Pacientes de } \\
\text { atención primaria } \\
\text { remitidos para } \\
\text { primaria consulta. }\end{array}$ & $\begin{array}{l}\text { Analiza la } \\
\text { concordancia } \\
\text { diagnostica } \\
\text { entre las dos } \\
\text { modalidades de } \\
\text { teleconsulta con } \\
\text { grupo control } \\
\text { de consulta } \\
\text { presencial de } \\
\text { dermatología. }\end{array}$ & $\begin{array}{l}\text { Demostrada la } \\
\text { fiabilidad de } \\
\text { la teleconsulta } \\
\text { destaca que la } \\
\text { TDTR consume } \\
\text { más recursos y } \\
\text { es más difícil de } \\
\text { coordinar sin } \\
\text { ser superior en } \\
\text { eficacia a la TDA. }\end{array}$ & SI & $1++$ \\
\hline $\begin{array}{l}\text { Pak, } \\
\text { Hon } \\
\text { S. }{ }^{24}\end{array}$ & $\begin{array}{l}2009 / \\
\text { EE.UU. }\end{array}$ & $\mathrm{N}=698$ & $\begin{array}{l}\text { Pacientes de } \\
\text { atención primaria } \\
\text { remitidos a } \\
\text { dermatológica } \\
\text { del Brooke army } \\
\text { medical center por } \\
\text { un periodo de } 4 \\
\text { meses en } 2006\end{array}$ & $\begin{array}{l}\text { Análisis de } \\
\text { minimización de } \\
\text { costos comparando } \\
\text { TDA con consulta } \\
\text { dermatológica } \\
\text { presencial. }\end{array}$ & $\begin{array}{l}\text { La TDA es en } \\
\text { promedio unos } 32 \\
\text { dólares menos } \\
\text { costosa por sujeto } \\
\text { estudiado. }\end{array}$ & SI & $1+$ \\
\hline $\begin{array}{l}\text { Van der } \\
\text { Heijden, } \\
\text { JP. }^{25}\end{array}$ & $\begin{array}{c}2011 \text { / } \\
\text { Holanda }\end{array}$ & $\begin{array}{l}1866 \text { Médicos; } \\
\text { realizándose un } \\
\text { total de } 37207 \\
\text { teleconsultas. }\end{array}$ & $\begin{array}{l}\text { Se valoran } \\
\text { fotografías } \\
\text { digitales enviadas } \\
\text { desde Atención } \\
\text { Primaria para } \\
\text { valoración diferida } \\
\text { por especialista } \\
\text { Marzo } 2007 \text { y } \\
\text { Septiembre } 2010 \text {. }\end{array}$ & $\begin{array}{l}\text { Análisis de } \\
\text { eficiencia, calidad } \\
\text { y costes. }\end{array}$ & $\begin{array}{l}\text { La TD es eficiente( } \\
\text { disminuye } 74 \% \text {, } \\
\text { de consultas a } \\
\text { especializada) de } \\
\text { calidad (solo se } \\
\text { precisa } 2 .^{\text {a }} \text { consulta } \\
\text { en un } 24 \% \text { ) y } \\
\text { disminuye costes } \\
\text { en un } 18 \%\end{array}$ & SI & $1+$ \\
\hline $\begin{array}{l}\text { Rios-yuil, } \\
\text { J.M. }{ }^{26}\end{array}$ & $\begin{array}{c}2011 \text { / } \\
\text { Panamá }\end{array}$ & $\mathrm{N}=30$ & $\begin{array}{l}\text { Pacientes remitidos } \\
\text { el mes de } \\
\text { abril } 2011 \text { a una } \\
\text { reunión semanal } \\
\text { científico-docente }\end{array}$ & $\begin{array}{l}\text { Análisis de } \\
\text { concordancia entre } \\
\text { teleconsulta y } \\
\text { consulta presencial } \\
\text { realizada por } \\
\text { dermatólogos. }\end{array}$ & $\begin{array}{l}\text { La TD puede } \\
\text { ser utilizada con } \\
\text { seguridad en el } \\
\text { diagnostico si el } \\
\text { paciente no puede } \\
\text { acudir a la consulta. }\end{array}$ & SI & $1-$ \\
\hline $\begin{array}{l}\text { Börve, } \\
\text { Alexander. }^{27}\end{array}$ & $\begin{array}{c}2013 \text { / } \\
\text { Suiza }\end{array}$ & $\mathrm{N}=62$ & $\begin{array}{l}\text { Pacientes derivados } \\
\text { al servicio de } \\
\text { dermatología, } \\
\text { en los que se } \\
\text { consideró necesaria } \\
\text { la biopsia o } \\
\text { extirpación de la } \\
\text { lesión. }\end{array}$ & $\begin{array}{l}\text { Valorar exactitud } \\
\text { diagnostica con } \\
\text { una aplicación } \\
\text { desarrollada para } \\
\text { Smartphone. Deter- } \\
\text { minar concordancia } \\
\text { interobservador } \\
\text { entre dos teleder- } \\
\text { matocopistas y un } \\
\text { dermatoscopista } \\
\text { presencial, así } \\
\text { como evaluar la } \\
\text { calidad de la ima- } \\
\text { gen obtenida. }\end{array}$ & $\begin{array}{l}\text { Exactitud } \\
\text { diagnostica } \\
\text { FTF } 66,7 \% \text { TD1 } \\
(50,7 \%, \mathrm{p}=0,04) \text { TD2 } \\
(60,97, \mathrm{p}=0,52) \\
\text { La calidad de } \\
\text { la imagen fue } \\
\text { catalogada } \\
\text { entre suficiente } \\
\text { y excelente en } \\
\text { un } 94 \% \text { y un } 84 \% \\
\text { de los casos por los } \\
\text { respectivos TD. }\end{array}$ & NO & $1-$ \\
\hline
\end{tabular}

Martínez-García, S. et al. $2007^{28}$ revisan las teleconsultas realizadas desde octubre de 2004 a octubre del 2006 en un servicio Hospitalario, que tenían la finalidad de filtrar patología tumoral desde los centros de salud, con el fin de dar prioridad a la asistencia en el hospital.

El estudio se realiza únicamente sobre patología tumoral por ser lesiones únicas y aisladas.

La patología tumoral maligna que más frecuentemente se consultó fueron son las queratosis actínicas en 161 ocasiones, y los carcinomas basocelulares en 73 casos.

La patología tumoral benigna que fue diagnosticada por teleconsulta se distribuyó en 201 casos de queratosis seborreicas, y en 147 casos de nevus melanocíticos adquiridos. 
Solamente en 62 de las 917 consultas realizadas por este sistema no se pudo emitir un diagnóstico. De ellas en 25 la imagen estaba desenfocada.

El $58 \%$ de los casos asistieron a consulta física, de ellos un $90 \%$ para confirmar diagnóstico y un $10 \%$ para realizar biopsia.

Concluyen que la teleconsulta aplicada como método de despistaje de lesiones tumorales malignas es una técnica útil, que podría incorporarse a las consultas de dermatología como una herramienta más del trabajo diario.

Ferrándiz, L. et al. 2008 $^{29}$ realiza un estudio de evaluación económica de la consulta de TDA en el Hospital Virgen Macarena (Sevilla); que conecta los Centros de Atención Primaria con la consulta de Dermatología hospitalaria, para la valoración de lesiones sospechosa de cáncer de piel. Se evaluaron los costes de las consultas atendidas desde Marzo de 2005 hasta Febrero de 2006 incluyendo aquellos pacientes con lesión sugerente de malignidad y susceptibles de tratamiento quirúrgico, se reclutaron 134 pacientes, con una media de edad de 70,25 años.

Se analizan datos sobre coste de la TD en comparación con la consulta convencional y de coste-efectividad atendiendo a la demora de espera prequirúrgica.

Como conclusiones señalan que la TDA, en este tipo de pacientes, es económicamente ventajosa y coste efectiva: 1,78 veces más barata (156,40 euros/paciente) que con la asistencia convencional (278,42 euros), se reducen costes en relación a los desplazamientos, número de consultas, acortamiento del tiempo de espera prequirúrgica; repercutiendo en una mejora de la calidad de la atención y de la satisfacción percibida por el paciente.

La sensibilidad y especificidad de la exploración dermatológica mediante telemedicina es estudiada por Baumeister, Thomas et al. $2009^{30}$, que en su trabajo buscan determinar si los resultados obtenidos con TD son igual de sensibles y específicos para el diagnóstico de lesiones cutáneas; como el examen directo en pacientes de ámbito laboral.

La muestra aleatorizada se compone de 100 trabajadores de una empresa de fabricación de automóviles en contacto con fluidos de corte;

El examen se realiza en horario laboral por un médico formado en el tema. El estudio incluyo la realización de una entrevista, junto a un cuestionario sobre condiciones laborales y el examen físico. Se categorizaron las lesiones de piel en 4 categorías: sin cambios, discretos cambios, moderados y severos. Se tomaron fotografías digitales, que fueron examinadas a posteriori por especialista en Dermatología.

Para determinar si existían diferencias significativas entre los dos métodos de exploración se utilizaron test apareados y pruebas no paramétricas (test Wilcoxon), no hallando diferencias estadísticamente significativas: $\mathrm{p}<0.001$.

Las conclusiones confirman la sensibilidad del examen mediante TDA para la detección de lesiones cutáneas en entorno laboral. La escasez de estudios que reflejen el papel de la TD en la salud ocupacional y su interés para la detección precoz de lesiones cutáneas de origen laboral.

El estudio de Vallejos, Quirina M. et al. 2009 ${ }^{31}$ evalúa si las interconsultas dermatológicas mediante TD pueden ayudar a profesionales de la salud que atienden a trabajadores agrícolas en clínicas rurales.

Para ello se evalúan 79 interconsultas, mediante TDA en trabajadores agrícolas que presentaron alguna enfermedad de la piel. El personal sanitario de las clínicas calificó un $12 \%$ de las consultas como algo útil, y el resto (88\%) de útiles o muy útiles. En un 13\% de los casos se modificaron los diagnósticos y en un $21 \%$ las orientaciones terapéuticas tras recibir el diagnóstico por TDA.

En base a estos resultados concluyeron que el acceso a servicios especializados (dermatólogos) es necesario para los centros de salud rurales y las consultas de TDA pueden ser una herramienta útil para satisfacer esta necesidad. 
La aplicación de la telefonía móvil a la TD es estudiada por Lamel S A, et al. $2012{ }^{32}$.

El objetivo del estudio fue determinar la concordancia entre la valoración presencial y mediante TD en pacientes incluidos en un programa de screening para cáncer de piel. Dos dermatólogos analizaron un total de 87 pacientes y 137 lesiones primero de forma o presencial y posteriormente, mediante TDA evaluaron fotografías de las mismas lesiones captadas a través de teléfono móvil. Los resultados analizados mediante índice de Kappa y test de McNemar mostraron valores de concordancia diagnóstica entre especialistas del $91,7 \%$ para la consulta tradicional; elevándose al 100\% para la TDA.

Se obtuvieron valores de concordancia del 81\% entre la valoración presencial y a través de TD mediante imágenes vía móvil, indicando un moderado acuerdo diagnóstico entre dermatólogos.

La telefonía móvil podría ser una innovadora técnica y útil herramienta para el screening de cáncer cutáneo en áreas o poblaciones donde no sea fácil el acceso a especialistas en dermatología.

Tabla V. Estudios transversales

\begin{tabular}{|c|c|c|c|c|c|c|c|}
\hline Autor & $\begin{array}{c}\text { Año / } \\
\text { País }\end{array}$ & Muestra & Colectivo & Efecto estudiado & Resultado & $\begin{array}{l}\text { Control } \\
\text { factor de } \\
\text { confusión }\end{array}$ & $\begin{array}{c}\text { Nivel de } \\
\text { evidencia }\end{array}$ \\
\hline $\begin{array}{l}\text { Martínez- } \\
\text { García, } \\
\text { S. }^{28}\end{array}$ & $\begin{array}{c}2007 \text { / } \\
\text { España }\end{array}$ & $\mathrm{N}=917$ & $\begin{array}{l}\text { Teleconsultas } \\
\text { realizadas entre } \\
\text { octubre de } 2004 \text { y } \\
\text { octubre de } 2006 \\
\text { en un servicio } \\
\text { hospitalario. }\end{array}$ & $\begin{array}{l}\text { Analiza a la } \\
\text { Teleconsulta } \\
\text { como método de } \\
\text { despistaste de } \\
\text { Lesiones malignas. }\end{array}$ & $\begin{array}{l}\text { La mayoría de } \\
\text { las lesiones } \\
\text { corresponden a } \\
\text { patología benigna. } \\
\text { El } 58 \% \text { fueron } \\
\text { citados para } \\
\text { consulta presencial. }\end{array}$ & NO & 3 \\
\hline $\begin{array}{l}\text { Ferrándiz, } \\
\text { L. }^{29}\end{array}$ & $\begin{array}{c}2008 \text { / } \\
\text { España }\end{array}$ & $\mathrm{N}=134$ & $\begin{array}{l}\text { Pacientes captados } \\
\text { con lesión de } \\
\text { piel sugerente } \\
\text { malignidad y } \\
\text { susceptible de } \\
\text { cirugía en consulta } \\
\text { de Primaria, } \\
\text { incluidos en } \\
\text { programa de TD } \\
\text { entre primaria } \\
\text { y especializada. } \\
\text { Periodo: Marzo } \\
\text { de } 2005 \text { - Febrero } \\
\text { de } 2006 \text {. }\end{array}$ & $\begin{array}{l}\text { Analizar datos de } \\
\text { coste de la TD y } \\
\text { coste -efectividad } \\
\text { medida por la } \\
\text { demora desde } \\
\text { teleconsulta } \\
\text { hasta la cirugía, } \\
\text { comparándolo } \\
\text { con consulta } \\
\text { convencional. }\end{array}$ & $\begin{array}{l}\text { TD es } \\
\text { económicamente } \\
\text { ventajosa y coste- } \\
\text { efectiva ( } 1,78 \text { veces } \\
\text { más barata), ahorro } \\
\text { desplazamientos, } \\
\text { número de } \\
\text { consultas y } \\
\text { disminuye } \\
\text { tiempo de espera } \\
\text { prequirúrgica. } \\
\text { La TD mejora } \\
\text { calidad atención } \\
\text { y satisfacción del } \\
\text { paciente. }\end{array}$ & SI & 3 \\
\hline $\begin{array}{l}\text { Baumeis- } \\
\text { ter, T. }\end{array}$ & $\begin{array}{c}2009 / \\
\text { Alemania }\end{array}$ & $\mathrm{N}=100$ & $\begin{array}{l}\text { Trabajadores } \\
\text { de industria del } \\
\text { metal en contacto } \\
\text { con fluidos } \\
\text { pertenecientes } \\
\text { a una empresa } \\
\text { automovilística } \\
\text { Mayo } 2007 .\end{array}$ & $\begin{array}{l}\text { Determinar si la TD } \\
\text { es igual de sensible } \\
\text { y específica para } \\
\text { el diagnóstico } \\
\text { de lesiones } \\
\text { cutáneas; como el } \\
\text { examen directo } \\
\text { convencional. }\end{array}$ & $\begin{array}{l}\text { Buena sensibilidad } \\
\text { de la TD para } \\
\text { diagnostico lesión } \\
\text { es cutáneas } \\
\text { e interés en } \\
\text { diagnostico precoz } \\
\text { dermatitis origen } \\
\text { laboral } \\
\text { Escasez de estudios } \\
\text { que valoren papel } \\
\text { de la TD a nivel de } \\
\text { Salud Laboral. }\end{array}$ & SI & 3 \\
\hline $\begin{array}{l}\text { Vallejos, } \\
\text { Quirina } \\
\text { M. }{ }^{31}\end{array}$ & $\begin{array}{l}2009 / \\
\text { EE.UU. }\end{array}$ & $\mathrm{N}=79$ & $\begin{array}{l}\text { Trabajadores del } \\
\text { campo que cum- } \\
\text { plían los criterios } \\
\text { de inclusión para } \\
\text { el estudio en } \\
\text { cuatro clínicas en } \\
\text { el este de Carolina } \\
\text { del Norte. }\end{array}$ & $\begin{array}{l}\text { Análisis explora- } \\
\text { torio sobre si la } \\
\text { teledermatología } \\
\text { puede ayudar a } \\
\text { satisfacer las nece- } \\
\text { sidades diagnosti- } \\
\text { cas de médicos de } \\
\text { clínicas rurales. }\end{array}$ & $\begin{array}{l}\text { Queda demostrada } \\
\text { la utilidad y la } \\
\text { satisfacción de } \\
\text { la TD para el } \\
\text { personal sanitario } \\
\text { de áreas rurales. }\end{array}$ & SI & 3 \\
\hline
\end{tabular}




\begin{tabular}{|c|c|c|c|c|c|c|c|}
\hline Autor & $\begin{array}{l}\text { Año / } \\
\text { País }\end{array}$ & Muestra & Colectivo & Efecto estudiado & Resultado & $\begin{array}{l}\text { Control } \\
\text { factor de } \\
\text { confusión }\end{array}$ & $\begin{array}{c}\text { Nivel de } \\
\text { evidencia }\end{array}$ \\
\hline $\begin{array}{l}\text { Lamel, } \\
\text { Sonia } \\
\text { A. }{ }^{32}\end{array}$ & $\begin{array}{l}2012 \text { / } \\
\text { EE.UU. }\end{array}$ & $\begin{array}{l}\text { N=87 (137 } \\
\text { lesiones } \\
\text { cutáneas) }\end{array}$ & $\begin{array}{l}\text { Pacientes incluidos } \\
\text { en programa } \\
\text { de cribado de } \\
\text { cáncer de piel. } \\
\text { Valoran consulta } \\
\text { convencional } \\
\text { e imágenes } \\
\text { transferidas por } \\
\text { telefonía móvil } \\
\text { para TD. }\end{array}$ & $\begin{array}{l}\text { Valorar } \\
\text { concordancia } \\
\text { entre consulta } \\
\text { convencional y } \\
\text { TD, concordancia } \\
\text { interobservador. }\end{array}$ & $\begin{array}{l}\text { Concordancia } \\
\text { interobservador } \\
\text { en consulta } \\
\text { convencional } \\
(91,7 \%) \text {, con TD } \\
(100 \%) \\
\text { Concordancia } 82 \\
\% \text { comparando } \\
\text { convencional y TD } \\
\text { Concordancia TD } \\
\text { convencional y } \\
\text { TD con imágenes } \\
\text { via telefonía móvil } \\
\text { (55\%-100\%). }\end{array}$ & SI & 3 \\
\hline
\end{tabular}

\section{DISCUSIÓN Y CONCLUSIONES}

Pese al avance de la telemedicina y la teledermatología, sólo hemos encontrado en nuestra búsqueda 4 referencias relacionadas con el mundo laboral y de ellas, dos no respondían a los objetivos de este estudio.

Los artículos referidos a exactitud diagnóstica ${ }^{20,21,26,27,30}$, demuestran una exactitud para la TD entre el 59 y el $89 \%{ }^{20}$.

En aquellos artículos que evalúan la exactitud diagnóstica de la consulta presencial comparada con la histopatología, parece ser más exacta la consulta presencial, seguida de la consulta síncrona y la asíncrona. Esto podría deberse a que el dermatólogo que realiza la visita presencial, considera otras características de la lesión no objetivables mediante TD, tales como consistencia de la lesión o búsqueda de otras lesiones. Así mismo, la teleconsulta en tiempo real permite la interacción con el paciente, no existiendo en la consulta diferida. De aquí la importancia de recomendaciones en cuanto a procedimentar las consultas de teledermatología no sólo en cuanto a toma de las imágenes sino en orden a registrar una buena historia clínica que incluya antecedentes personales, cronología de las lesiones, tratamientos utilizados y que en nuestro caso debería acompañarse también de una exhaustiva historia laboral

En los últimos años, con el envío de imágenes dermatoscópicas a través de aplicaciones móviles, se consigue mejorar la exactitud diagnóstica.

En el caso de despistaje de lesiones tumorales aumenta el porcentaje valoración posterior en consulta tradicional, dada la importancia del subdiagnóstico en este tipo de lesiones. Sin embargo, el uso de la TD posibilita la disminución de los tiempos de espera tanto para diagnóstico como para tratamiento, con mejora de la satisfacción percibida por parte de los usuarios ${ }^{28,29,32}$.

Otro dato a tener en cuenta es que en muchos de los estudios, tanto la consulta presencial como la teleconsulta, ha sido realizada por dermatólogos experimentados. Serían precisos estudios en los que la consulta presencial se realizase por un médico del trabajo, para evaluar correctamente la exactitud diagnóstica en el medio laboral.

Cuando hablamos de eficiencia ${ }^{25}$ y utilidad de la teleconsulta para servicios no especializados (médicos de atención primaria); los datos revelan una disminución del número de derivaciones, resultando útil o muy útil en el $88 \%$ de los $\operatorname{casos}^{32}$; ayudando al diagnóstico y manejo del paciente.

No se encuentran diferencias estadísticamente significativas en cuanto a la sensibilidad para la detección de lesiones cutáneas entre la TD y la consulta presencial, siendo beneficioso dentro de la patología laboral para el diagnóstico precoz y el tratamiento inmediato ${ }^{30}$. 
En relación a los costes, todos los artículos muestran acuerdo respecto a los beneficios que demuestra la TD, pese a que los parámetros estudiados pueden demostrar amplia variabilidad ${ }^{16-23-24-25-29}$. Este ahorro se refleja en la disminución de desplazamientos y de tiempos de espera o pérdida de productividad, lo que repercute en la calidad del servicio y el grado de satisfacción percibida por el paciente.

La satisfacción y preferencias de los pacientes son similares ${ }^{20-29}$, pero dado el impacto que presentan las lesiones dermatológicas en la calidad de vida percibida, muchos pacientes prefieren la TD dada la reducción del tiempo de espera que supone tanto para el diagnóstico como para el tratamiento.

En un alto porcentaje de pacientes (79\%-86\%) la satisfacción era elevada con el uso de la TD $^{21}$. Entre los facultativos usuarios de la TD el nivel de satisfacción va desde un $21 \%$ al $92 \%$ entre los médicos que solicitan la teleconsulta, dependiendo sobre todo si se tratan de dermatólogos o médicos con otra especialidad y entre un $72,5 \%$ y $92 \%$ entre los dermatólogos que la reciben ${ }^{20}$.

La mayoría de los artículos hacen referencia a la fiabilidad y validez diagnóstica entre dermatólogos ${ }^{31}$, pero probablemente ésta sería mucho más evidente si los estudios se realizaran entre médicos del trabajo y dermatólogos. Las lesiones dérmicas son una parte importante dentro de la patología laboral, pero la formación dermatológica no es a veces la suficiente entre los médicos del trabajo, siendo ellos, sin embargo, los mejores conocedores de las condiciones del trabajo y su posible repercusión en este tipo de patologías.

Podemos concluir, por tanto, en base a los datos obtenidos con nuestra revisión y recordando los objetivos específicos que determinamos al inicio de nuestra exposición los siguientes aspectos.

La TD resulta una herramienta diagnóstica precisa con una exactitud equiparable a la consulta dermatológica convencional. (NIVEL EVIDENCIA 1-)

Se necesitarían más trabajos para demostrar que dicha exactitud diagnóstica podría ser más elevada en el caso de tratarse de un médico del trabajo realizando una consulta de TD en el ámbito laboral.

La TD resulta coste beneficiosa y coste-efectiva, aumentando en aquellos casos en que se tienen en cuenta factores críticos tales como: distancia del paciente a la consulta física; pérdida de productividad, reducción de tiempos de espera para diagnóstico e inicio del tratamiento. Todos ellos factores claves a considerar en la práctica de la Medicina del Trabajo. (NIVEL EVIDENCIA 1+)

Los pacientes se muestran satisfechos con la consulta de TD, reflejándose en una mejor aceptación de esta modalidad de consulta sobre todo cuando conlleva una disminución del tiempo de espera para la valoración por especialista. (NIVEL EVIDENCIA 1+)

El grado de satisfacción del profesional usuario de la TD se constata tanto a nivel de los profesionales de atención primaria como de los dermatólogos que intervienen en ellas. (NIVEL EVIDENCIA 1+)

Consideramos, por tanto, que la implementación de esta nueva herramienta en la práctica del Médico del trabajo sería de gran utilidad, dada la frecuencia e impacto de las patologías dermatológicas. La TD permitiría agilizar el diagnóstico, iniciar precozmente un tratamiento y una disminución de los costes indirectos, evitando ausencias prolongadas del puesto de trabajo y pérdidas en la productividad laboral, y una mayor calidad de vida en el trabajador con una dermatopatía.

Se plantea, por tanto, un reto para la Medicina del Trabajo ante la necesidad de formación a los especialistas en el manejo de la Teledermatología y sus distintas modalidades, para mejorar un área de importante repercusión en el campo de la salud laboral como es la dermatología ocupacional. 


\section{REFERENCIAS BIBLIOGRÁFICAS}

1. WHO (World Health Organization). Telemedicine. Opportunities and developments in member states. Report on the second global survey on eHealth. Global Observatory for eHealth series. Volume 2. ISBN 9789241564144 ISSN 2220-5462@ World Health Organization 2010. Disponible en: http://www. who.int/goe/publications/goe_telemedicine_2010.pdf.

2. American Telemedicine Association. Learn about Telemedicine. http://www.americantelemed.org/learn.

3. WHO/E-Health. http://www.who.int/trade/glossary/story021/en/.

4. Digital Agende for Europe- European Commission- Internet Explorer. https://ec.europa.eu/digital-agenda/ en/telemedicine.

5. Trevor Lewis, Christina Synowiec, Gina Lagomarsino\& Julian Schweitzer. E-health in low- and middleincome countries: findings from the Center for Health Market Innovations. Bulletin of the WHO. February 2012

6. Digital Agende for Europe- European Commission- Internet Explorer. http://ec.europa.eu/information society/apps/projects/factsheet/index.cfm?project_ref=25048.

7. Nexes Final Workshop http://www.nexeshealth.eu/.

8. American Telemedicine Association. Quick Guide to Store-Forward and Live-Interactive Teledermatology for Referring Providers April 2012.

9. American Telemedicine Association. Practice Guideline For Teledermatology. December 2007.

10. Moreno-Ramírez D, Ferrándiz L, Pérez AM, Carrasco R, Rios JJ, Camacho F. Teledermatology as a filtering system in pigmented lesion clinics.J Telemed Telecare.2005;11:298-303.

11. Oliveira MR, Wen CL, Neto CF, Silveira PS, Rivitti EA, Bohm GM Web site for training nonmedical healthcare workers to identify potentially malignant skin lesions and for teledermatology. Telemed J E Health 2002;8:323-32.

12. Moreno-Ramírez D, Ferrándiz L, Nieto-García A, Carrasco R, Moreno-Álvarez P, Galdeano R, et al. Storeand-forward teledermatology in skin cancer triage: experience and evaluation of 2009 teleconsultations. Arch Dermatol 2007;143:479-84.

13. Edison KE, Ward DS, Dyer JA, Lane W, Chance L, Hicks LL Diagnosis, diagnostic confidence, and management concordance in live interactive and store-and-forward teledermatology compared to in-person examination. Telemed J E Health 2008;14:889-95.

14. Taberner Ferrer R, Pareja Bezares A, Llambrich Mañes A; et al.Fiabilidad diagnóstica de una consulta de teledermatologia asincrona.Atencion Primaria.2009;41(10):552-557.

15. Observatorio de las contingencias profesionales de la Seguridad Social. Enfermedades profesionales (CEPROSS) http://www.segsocial.es/Internet_1/Estadistica/Est/Observatorio_de_las_Enfermedades_Profesionales/ cepross2k11/index.htm.

16. Observatorio de las contingencias profesionales de la Seguridad Social. Informe Anual 2012. Observatorio Enfermedades Profesionales (Cepross) y Enfermedades Causadas o Agravadas por el Trabajo (Panotrass).

17. Observatorio de las contingencias profesionales de la Seguridad Social. Estadísticas Ministerio Observatorio Enfermedades Profesionales. Informe Anual 2012.

18. Observatorio de las contingencias profesionales de la Seguridad Social. Estudio Epidemiológico Descriptivo de los Partes Cerrados Notificados a Cepross. Periodo 2007-2012. Ministerio de Empleo y Seguridad Social.

19. Scottish Intercollegiate Guidelines Network. SIGN 50: A guideline developer's handbook. http://www. sign.ac.uk. 2008.

20. Moreno-Ramírez D, Ferrándiz L, Nieto-García A, Villegas-Portero R. Teledermatology Med Clin (Barc) 2008 Apr 12;130(13):496-503.

21. Warshaw EM, Hillman YJ, Greer NL, Hagel EM, MacDonald R, Rutks IR, et al. Teledermatology for diagnosis and management of skin conditions: A systematic review J Am Acad Dermatol 2011;64(4):759-772.

22. Bowns IR, Collins K, Walters SJ, McDonagh AJ. Telemedicine in dermatology: a randomised controlled trial Health Technol Assess 2006 Nov;10(43):iii-iv, ix-xi, 1-39.

23. G Romero, M García, E Vera, C Martínez, P Cortina, P Sánchez, A Guerra. [Preliminary results of DERMATEL: prospective randomized study comparing synchronous and asynchronous modalities of teledermatology]. Actas dermo-sifiliográficas 2007;97(10):630-636.

24. Pak HS, Datta SK, Triplett CA, Lindquist JH, Grambow SC, Whited JD. Cost Minimization Analysis of a Store-and-Forward Teledermatology Consult System Telemedicine and e-Health 2009;15(2):160-165. 
25. Van der Heijden JP, de Keizer NF, Bos JD, Spuls PI, Witkamp L. Teledermatology applied following patient selection by general practitioners in daily practice improves efficiency and quality of care at lower cost Br J Dermatol 2011; 2011;165(5):1058-1065.

26. Ríos-Yuil JM. Correlación del Teleateneo con el Ateneo presencial de Dermatología en el diagnóstico de las patologías cutáneas Actas Dermo-Sifiliográficas 2012;103(2):138-143.

27. Börve A, Terstappen K, Sandberg C, Paoli J. Mobile teledermoscopy-there's an app for that! Dermatology Practical \& Conceptual 2013;3(2).

28. Martínez-García S, Del Boz-González J, Martín-González T, Samaniego-González E, Crespo-Erchiga V. Teledermatología. Revisión de 917 teleconsultas Actas Dermo-Sifiliográficas 2007;98(5):318-324.

29. Ferrándiz L, Moreno-Ramírez D, Ruiz-de-Casas A, Nieto-García A, Moreno-Álvarez P, Galdeano R, et al. Teledermatología prequirúrgica en pacientes con cáncer cutáneo no melanoma. Evaluación económica Actas Dermo-Sifiliográficas 2008;99(10):795-802.

30. Baumeister T, Weistenhöfer W, Drexler H, Kütting B. Prevention of work-related skin diseases: teledermatology as an alternative approach in occupational screenings Contact Derm 2009;61(4):224 -230.

31. Vallejos QM, Quandt SA, Feldman SR, Fleischer,,Alan B.,Jr, Brooks T, Cabral G, et al. Teledermatology Consultations Provide Specialty Care for Farmworkers in Rural Clinics The Journal of Rural Health 2009;25(2):198-202.

32. Lamel SA, Haldeman KM, Ely H, Kovarik CL, Pak H, Armstrong AW. Application of mobile teledermatology for skin cancer screening J Am Acad Dermatol 2012;67(4):576-581.

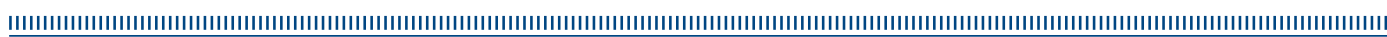

